\title{
Terapêutica biológica da asma grave em crianças e adolescentes
}

\section{Biological treatments for severe asthma in childhood and adolescence}

Data de receção / Received in: 19/1/2020 Data de aceitação / Accepted for publication in: | 8/5/2020

\author{
Rev Port Imunoalergologia 2021;29 (I):27-38
}

Pedro Botelho Alves', Iolanda Alen Coutinho', Ana Luísa Moura', Frederico S. Regateiro',2,3

I Serviço de Imunoalergologia, Centro Hospitalar Universitário de Coimbra

${ }^{2}$ Instituto de Imunologia, Faculdade de Medicina, Universidade de Coimbra

${ }^{3}$ Coimbra Institute for Clinical and Biomedical Reseach (iCBR), Faculdade de Medicina, Universidade de Coimbra

\section{RESUMO}

A asma é a doença inflamatória crónica mais comum na idade pediátrica. Entre as crianças e adolescentes com asma, cerca de 4,5\% apresentam asma grave. Nos últimos anos foram aprovados alguns anticorpos monoclonais para o tratamento da asma grave na idade pediátrica. Atualmente, os biológicos comercializados em Portugal para o tratamento da asma grave pediátrica são o omalizumab (anti-lgE, aprovado para crianças e adolescentes a partir dos 6 anos), o mepolizumab (anti-IL5, também a partir dos 6 anos) e o dupilumab (anti-IL4/IL-I3, a partir dos I2 anos). Todos os biológicos atualmente disponíveis são dirigidos a moléculas envolvidas na inflamação de tipo Th2, o tipo de inflamação mais frequente em asmáticos destas faixas etárias. Este artigo tem como objetivo rever a informação existente sobre as terapêuticas biológicas atualmente disponíveis ou em desenvolvimento para o tratamento da asma grave em idade pediátrica.

Palavras-chave: Asma grave, criança, adolescente, anticorpos monoclonais, IgE, IL-5, IL-4, IL-I3, endótipos de asma.

\section{ABSTRACT}

Asthma is the most common chronic inflammatory disease in pediatric ages. Among the children and adolescents with asthma, it is estimated that $4.5 \%$ present with severe asthma. Recently, several monoclonal antibodies were approved for the treatment of severe asthma in pediatric patients. Currently, the biologics approved for use in Portugal for the treatment of 
Pedro Botelho Alves, lolanda Alen Coutinho, Ana Luísa Moura, Frederico S. Regateiro

pediatric severe asthma are: omalizumab (anti-lgE, approved for children and adolescents aged 6 years and over), mepolizumab (anti-IL-5, also for patients 6 years and older), and dupilumab (anti-IL4/IL-I3, for patients aged I2 years and over). All the currently available biologics target molecules involved in Th2 inflammation, the most frequent type of inflammation in pediatric asthma patients. We here review the evidence on the biologics currently approved or under development for the treatment of pediatric severe asthma.

Keywords: Adolescent, child, endotypes, IgE, IL-5, IL-4, IL-I3, monoclonal antibodies, severe asthma.

\section{INTRODUÇÃO}

A asma é a doença inflamatória crónica mais comum na idade pediátrica'. Clinicamente é caraterizada por episódios recorrentes de sibilância, tosse de predomínio noturno, dispneia e opressão torácica $^{2,3}$. A maioria das crianças com sibilância recorrente em idade pré-escolar apresenta resolução do quadro clínico antes da adolescência, sendo o risco de evolução para asma persistente dependente do fenótipo de sibilância. Classicamente, os fenótipos de sibilância recorrente dividem-se em sibilância transitória, sibilância persistente e sibilância de início tardio ${ }^{4-7}$. Um estudo nacional, publicado em 2014 por Pité et al., identificou três fenótipos de sibilância em crianças: sibilância persistente atópica, sibilância persistente não atópica e sibilância transitória não atópica $^{8}$. No seguimento a 13 anos, verificou-se que as crianças com sibilância persistente atópica, caracterizada pela presença de atopia e história pessoal de rinite em idade pré-escolar, têm significativamente maior risco de persistência clínica e evolução para asma ${ }^{8}$.

A classificação da asma em fenótipos, endótipos, graus de gravidade e de controlo permite uma abordagem terapêutica mais dirigida e eficaz. Os fenótipos de asma são baseados em dados clínicos (e.g., idade de apresentação, fatores de agravamento, comorbilidades, resposta à terapêutica, etc). Porém, estas características clínicas relacionam-se apenas parcialmente com o tipo de inflamação presente nas vias aéreas das crianças asmáticas. Por outro lado, a classificação em endótipos é baseada nos mecanismos fisiopatológicos subjacentes, sendo de- finidos por padrões celulares e/ou moleculares, e poderá explicar as características fenotípicas ${ }^{9}$. Pelo menos em teoria, a classificação em endótipos permitirá uma melhor escolha terapêutica ${ }^{10}$. Atualmente, os dois endótipos mais consensuais são baseados na presença de inflamação Th2 (Th2-high vs Th2-low). Na inflamação Th2, as alarminas epiteliais TSLP, IL-25 e IL-33 estimulam a produção de citocinas IL-4, IL-5 e IL-I3 pelas células dendríticas e células linfoides inatas tipo 2 (ILC-2). Estas citocinas vão induzir a diferenciação de linfócitos Th2 e a proliferação, ativação e acumulação de eosinófilos no epitélio brônquico e, em alguns doentes, a produção de IgE específica "'.

A definição de fenótipos de asma grave na idade pediátrica é um processo complexo e pouco consensual, em parte devido às limitações para investigação clínica neste grupo etário e ao reduzido número de estudos. Um dos trabalhos mais reconhecidos nesse campo identificou quatro clusters, de gravidade crescente, num grupo de 16I crianças seguidas no Severe Asthma Research Program (SARP): I - asma sintomática de início tardio, que apresentou menor gravidade, presente em 48 (29,8\%) doentes; 2 - asma atópica de início precoce com função pulmonar normal, em 52 (32,3\%); 3 - asma atópica de início precoce com limitação moderada do fluxo aéreo, em 32 (19,9\%); 4 - asma atópica de início precoce com limitação significativa do fluxo aéreo, em 29 (I8\%). Os clusters 3 e 4 - mais graves - apresentaram níveis superiores de FeNO, bem como mais comorbilidades e maior necessidade de tratamento com corticoterapia oral ${ }^{12}$.

Uma limitação importante desde trabalho foi o facto de nenhum dos clusters corresponder devidamente às 
definições de asma grave da ATS ou do GINA, que foram extrapoladas a partir da idade adulta. Estes dados reforçam a heterogeneidade marcada da asma grave em crianças, também verificada nos adultos. Contudo, ao contrário dos adultos com asma de início tardio, a asma grave com início em idade pediátrica parece predominantemente associada a um endótipo Th2-high e a mecanismos alérgicos - eosinofilia periférica, maior sensibilidade a aeroalergénios, concentrações elevadas de lgE e elevações persistentes de $\mathrm{FeNO}^{13}$.

A investigação em transcriptómica, proteómica e metabolómica de doentes com asma tem procurado identificar outros endótipos e genes relacionados, assim como estabelecer associações entre endótipos e fenótipos clínicos de asma ${ }^{10}$. A caracterização endotípica da asma assume particular relevância no contexto da asma grave. O conceito de asma grave, de acordo com definição da Global Initiative for Asthma (GINA) 2019, está intimamente relacionado com a resposta à terapêutica e compreende um dos dois seguintes critérios: I - asma não controlada com terapêutica máxima otimizada nos steps 4 ou 5 (ou step 4 em crianças com idade inferior a 6 anos); 2 - asma que agrava com a redução destes steps terapêuticos ${ }^{14}$. Também a definição da European Respiratory Society (ERS) e da American Thoracic Society (ATS) reforça a necessidade de terapêutica inalatória em alta dose e, possivelmente, de corticoterapia sistémica. Estima-se que cerca de $4,5 \%$ das crianças asmáticas têm asma grave ${ }^{15,16}$.

$\mathrm{Na}$ asma grave, é frequente o recurso à corticoterapia oral e/ou inalada (ICS) em alta dose, o que pode ter efeitos indesejados, devido à potencial supressão do eixo hipotálamo-hipófise (embora a insuficiência adrenal seja rara), alteração comportamental (esquemas de corticoterapia oral em exacerbações de asma podem causar ansiedade, mania, irritabilidade ou comportamentos agressivos) ou interferência no metabolismo ósseo (possível redução da densidade mineral óssea com doses de ICS elevadas e a longo prazo), entre outros efeitos ${ }^{17}$. Em particular, o uso de corticosteroides orais tem um efeito dose-dependente no atraso do crescimento das crianças, com possível impacto negativo na altura final. Com os ICS em alta dose, os dados são menos conclusivos. $O$ efeito dos ICS no crescimento de crianças em idade pré-pubertária é mais pronunciado com maiores doses de fármaco (superiores a dose-equivalente de $400 \mu \mathrm{g}$ de beclometasona), mas ocorre principalmente durante o primeiro ano de tratamento, sendo incerto que este atraso tenha impacto na estatura da idade adulta. Deste modo, é importante encontrar alternativas terapêuticas que reduzam o uso destes fármacos e os seus potenciais efeitos adversos ${ }^{17,18}$. A asma grave na idade pediátrica pode também causar inflamação e remodeling das vias aéreas, o que potencialmente leva a obstrução persistente do fluxo aéreo e a um declínio marcado do $\mathrm{FEV}_{\text {, }}$ na idade adulta $^{19}$.

Vários medicamentos biológicos dirigidos a moléculas da inflamação Th2 foram aprovados para o tratamento da asma moderada a grave em adultos ${ }^{20}$. Os ensaios clínicos e guidelines terapêuticas ${ }^{14}$ distinguem entre adultos (geralmente, acima dos 18 anos), adolescentes (entre os 12 e os 18 anos) e crianças (abaixo dos 12 anos). $O$ GINA considera ainda a idade pré-escolar (abaixo dos 6 anos), que apresenta especificidades no diagnóstico e tratamento. $\mathrm{Na}$ ausência de ensaios clínicos dirigidos apenas a faixas etárias pediátricas, os resultados dos estudos clínicos em adultos para asma grave têm sido, frequentemente, extrapolados para adolescentes, dada a putativa semelhança fisiopatológica. Em idades mais precoces, as diferenças são mais significativas, quer na fisiologia das crianças, quer na fisiopatologia da doença, estando disponível menor evidência científica nestes grupos etários ${ }^{21}$. Atualmente não existem biológicos aprovados para o tratamento da asma grave em crianças com menos de 6 anos.

Este artigo tem como objetivo rever a informação existente sobre as terapêuticas biológicas atualmente disponíveis ou em desenvolvimento para o tratamento da asma grave em crianças e adolescentes (Quadro I e Figura I). 
Quadro I. Resumo dos principais estudos concluídos sobre as terapêuticas biológicas em comercialização/investigação para a asma grave em idade pediátrica. Foram excluídos estudos ainda a decorrer ou suspensos

\begin{tabular}{|c|c|c|c|c|}
\hline Estudo & Anticorpo & $\begin{array}{l}\text { Doentes } \\
\left(\mathrm{n} .^{\circ}\right)\end{array}$ & $\begin{array}{l}\text { Idades } \\
\text { (anos) }\end{array}$ & Resultados publicados \\
\hline Lanier et al. ${ }^{27}$ & Omalizumab & 627 & $6-11$ & $\begin{array}{l}\text { Taxa anualizada de exacerbações - redução de } 43 \% \text { vs. placebo } \\
N .^{\circ} \text { exacerbações com hospitalização - redução de } 50 \% \text { vs. placebo }\end{array}$ \\
\hline ICATA $^{26}$ & Omalizumab & 419 & $6-20$ & $\begin{array}{l}\text { N. }{ }^{\circ} \text { dias com sintomas - redução de } 24,5 \% \text { vs. placebo } \\
\text { Taxa de exacerbações - redução de } 18 \% \text { vs. placebo } \\
\text { Redução da dose de corticosteroides inalados }\end{array}$ \\
\hline PROSE $^{29}$ & Omalizumab & 478 & $6-17$ & $\begin{array}{l}\text { Taxa de exacerbações - } 11,3 \% \text { vs. } 21,0 \% \text { placebo; diferença não } \\
\text { significativa vs. aumento de corticosteroides inalados } \\
\text { Maior benefício no período run-in }\end{array}$ \\
\hline STELLAIR 32 & Omalizumab & 872 & $\geq 6$ & $\begin{array}{l}78,5 \% \text { de crianças tiveram redução }>40 \% \text { da taxa de exacerbações } \\
\text { Eficácia semelhante em subgrupos com elevado ou reduzido nú- } \\
\text { mero de eosinófilos periféricos }\end{array}$ \\
\hline DREAM ${ }^{36}$ & Mepolizumab & 621 & $12-74$ & $\begin{array}{l}\text { Taxa de exacerbações - redução de } 39-52 \% \text { vs. placebo } \\
\text { Redução maior em doentes com maior eosinofilia periférica }\end{array}$ \\
\hline MENSA $^{37}$ & Mepolizumab & 576 & $12-82$ & $\begin{array}{l}\text { Taxa de exacerbações - redução de } 47-53 \% \text { vs. placebo } \\
\text { FEVI médio - aumento de } 98-100 \mathrm{ml} \text { vs. placebo } \\
\text { Melhoria nos scores de qualidade de vida e controlo da asma }\end{array}$ \\
\hline SIRIUS ${ }^{38}$ & Mepolizumab & 135 & $16-74$ & $\begin{array}{l}\text { Dose de corticosteroides sistémicos - redução de } 50 \% \text { vs. placebo } \\
\text { Taxa anualizada de exacerbações - redução de } 32 \% \text { vs. placebo } \\
\text { Melhoria no score ACQ-5 de controlo da asma }\end{array}$ \\
\hline $\begin{array}{l}\text { COSMOS }^{39} \\
\text { (extensão MENSA/ } \\
\text { SIRIUS) }\end{array}$ & Mepolizumab & 651 & $12-82$ & $\begin{array}{l}\text { Reações adversas: sistémicas - } 2 \% \text {; local de injeção - } 4 \% \\
\text { Manutenção da redução da taxa de exacerbações e dose de cor- } \\
\text { ticosteroide sistémico em } 52 \text { semanas de tratamento }\end{array}$ \\
\hline $\begin{array}{l}\text { COLUMBA }^{40} \\
\text { (extensão DREAM) }\end{array}$ & Mepolizumab & 347 & $12-74$ & $\begin{array}{l}\text { Efeitos adversos atribuídos ao tratamento - } 28 \% \text { (cefaleias }-4 \% \text {; } \\
\text { reações no local de injeção - } 12 \% \text { ) } \\
\text { Manutenção da redução da taxa de exacerbações e score ACQ-5 } \\
\text { em mais de } 155 \text { semanas de tratamento }\end{array}$ \\
\hline Gupta et $a l^{41}$ & Mepolizumab & 30 & $6-11$ & $\begin{array}{l}\text { Redução do número de eosinófilos circulantes, taxa de exacerba- } \\
\text { ções e controlo sintomático } \\
\text { Eventos adversos: em geral - } 90 \% \text {; graves - } 23 \% \text { ( } 0 \% \text { atribuídos ao } \\
\text { tratamento) }\end{array}$ \\
\hline BREATH $* 43$ & Reslizumab & 39 & $12-17$ & $\begin{array}{l}\text { Taxa de exacerbações em adolescentes - não redução (razão } \\
\text { [reslizumab/placebo] de 2,09) }\end{array}$ \\
\hline $\begin{array}{l}\text { SIROCCO/ } \\
\text { CALIMA }^{*, 44}\end{array}$ & Benralizumab & 108 & $12-17$ & $\begin{array}{l}\text { Taxa de exacerbações em adolescentes - não redução ( } 70 \% \text { vs. } \\
41 \% \text { placebo }\end{array}$ \\
\hline QUEST ${ }^{47}$ & Dupilumab & 1902 & $\geq 12$ & Taxa anualizada de exacerbações - redução de $65,8 \%$ vs. placebo \\
\hline VENTURE $^{50}$ & Dupilumab & 210 & $\geq 12$ & $\begin{array}{l}\text { Dose de corticosteroides sistémicos - redução de } 70,1 \% \text { vs. } \\
41,9 \% \text { placebo }\end{array}$ \\
\hline
\end{tabular}

* Programa de 4 estudos - representado na tabela subgrupo pediátrico de um total de 1759 doentes; $¥$ Representado na tabela subgrupo pediátrico de um total de 2295 doentes. 


\begin{tabular}{|c|c|c|c|c|}
\hline Alvo & $0-6$ anos & 6-12 anos & 12-18 anos & $>18$ anos \\
\hline \multicolumn{5}{|c|}{ Aprovados } \\
\hline IgE & & \multicolumn{3}{|l|}{ OMALIZUMAB } \\
\hline IL-5 & & \multicolumn{3}{|c|}{ MEPOLIZUMAB } \\
\hline IL-5 & & & & RESLIZUMAB \\
\hline IL-5R $\alpha$ & & & & BENRALIZUMAB \\
\hline IL-4/13 & & & \multicolumn{2}{|l|}{ DUPILUMAB } \\
\hline \multicolumn{5}{|c|}{ Em desenvolvimento } \\
\hline TSLP & & & & TEZEPELUMAB \\
\hline
\end{tabular}

Figura I. Anticorpos monoclonais aprovados ou em fases de desenvolvimento avançado para o tratamento da asma grave. Para cada biológico é apresentado o seu alvo terapêutico (coluna da esquerda) e as faixas etárias para as quais estão aprovados ou em desenvolvimento

\section{TERAPÊUTICA ANTI-IgE}

\section{Omalizumab}

O omalizumab é um anticorpo monoclonal lgGI de administração subcutânea com atividade anti-lgE que se fixa à porção $\mathrm{C} \varepsilon 3$ da lgE livre, impedindo, deste modo, a ligação da IgE aos recetores FcદRI na superfície de mastócitos, basófilos e outras células do sistema imunitário ${ }^{22}$.

O omalizumab foi aprovado pela FDA em 2003 para o tratamento da asma moderada a grave em doentes com 12 ou mais anos e em 2005 pela EMA para o tratamento de doentes com asma alérgica não controlada com múltiplas exacerbações apesar do tratamento com doses altas de corticoides inalados/ $\beta 2$-agonistas de longa ação e com $\mathrm{FEVI}<80 \%$ do previsto. A utilização em doentes com idades a partir dos 6 anos foi aprovada pela EMA em 2009 e pela FDA em 201623,24. O omalizumab apresenta um elevado perfil de segurança em crianças, adolescentes e adultos, com cefaleias e reações no local de injeção a serem os efeitos indesejáveis mais observa$\operatorname{dos}^{24,25}$. As doses de administração do fármaco variam entre 75 e $375 \mathrm{mg}$ (para idades entre os 6 e os II anos) e 150 a $375 \mathrm{mg}$ (para idades iguais ou superiores a 12 anos) a cada 2 ou 4 semanas. A determinação da posologia e da frequência recomendadas depende dos níveis de lgE sérica total medidos antes do início do tratamento e do peso corporal do doente. Com o crescimento, poderá haver variação do peso e ser necessário ajustar a dose de manutenção com base em tabelas fornecidas pela empresa produtora, que discriminam categorias entre os 20 e os $150 \mathrm{~kg}^{24}$.

Foram publicados vários ensaios clínicos randomizados controlados com placebo ${ }^{26-29}$ e vários estudos observacionais ${ }^{30,31}$ para avaliar a eficácia e segurança do omalizumab em asmáticos em idade pediátrica. Entre os ensaios clínicos destaca-se o estudo de Lanier et al. ${ }^{27}$, que incluiu 627 crianças com idades entre os 6 e os 12 anos com asma alérgica mal controlada, em que se verificou que o tratamento com omalizumab durante um ano reduziu a taxa de exacerbações em $43 \%$ e de exacerbações graves e hospitalizações em $50 \%$ versus placebo ${ }^{27}$. No estudo ICATA (Busse et al.), com 419 doentes entre os 6 e os 20 anos com asma alérgica não controlada, constatou-se que a adição de omalizumab à terapêutica-base durante 60 semanas reduziu, versus placebo, o número de dias com sintomas em $24,5 \%$, a taxa de exacerbações em $18 \%$ e houve ainda uma redução da dose de ICS. Numa análise post hoc, os autores realçaram que o benefício nas exacerbações abrangeu o período da primavera e do inverno, ou seja, a época polínica e o período de maior frequência das infeções virais. Estes dados sugerem que o omalizumab poderá ter um papel não só na prevenção de exacerbações de causa alérgica mas também de outras causas, nomeadamente por infeções ${ }^{26}$. $O$ efeito preventivo de exacerbações com desencadeante viral é igualmente sugerido pelos resultados do estudo seguinte. Ao contrário do ICATA, foi aplicado no período verão-outono e poderá indicar um efeito independente da sazonalidade.

O estudo PROSE (Back to school - PROSE study) ${ }^{29}$ foi um estudo sazonal, em contexto de "regresso às aulas" após as férias de verão, realizado em crianças com idades entre os 6 e os 17 anos, com asma persistente não controlada, com $>200$ ug de fluticasona equivalente/dia e que tinham tido uma exacerbação tratada com corticosteroide sistémico nos últimos 19 meses. $O$ estudo incluiu três braços terapêuticos, a aplicar durante 90 dias, entre 4 a 
6 semanas antes do regresso às aulas: I) duplicação da dose habitual de ICS; 2) associação de omalizumab à terapêutica de manutenção habitual; ou 3) associação de placebo em vez de omalizumab. A associação de omalizumab à terapêutica reduziu a taxa de exacerbações quando comparada com o placebo (II,3\% vs $21,0 \%$; OR 0,48 ). A melhoria obtida com o omalizumab não foi significativamente diferente da obtida com o aumento da dose de ICS (8,4\% vs II, I\%; OR 0,73). O benefício do omalizumab foi particularmente observado nos doentes que tiveram exacerbações durante o período de run-in, tanto versus placebo $(6,4 \%$ vs $36,3 \%$; OR 0,12$)$ como versus a duplicação da dose de ICS (2,0\% vs 27,8\%; OR 0,05). Apesar de o omalizumab ter diminuído o número de episódios sintomáticos, a duração dos episódios não foi afetada. A resposta positiva ao omalizumab associou-se a uma meIhor produção de IFN- $\alpha$ em resposta a rinovírus, sugerindo que a terapêutica anti-lgE poderá ser benéfica também em exacerbações de asma desencadeadas por esta infeção viral ${ }^{29}$.

Em crianças com asma grave, uma resposta total e precoce ao omalizumab associou-se a FEV, normal, à presença de comorbilidades atópicas (sensibilizações múltiplas, dermatite atópica ativa e alergia alimentar) e a níveis elevados de eosinofilia sérica, lgE total e FeNO". Alguns dados recentes contestam a associação da resposta ao omalizumab com a eosinofilia: o estudo STELLAIR constatou que a eficácia do omalizumab em doentes adultos e pediátricos com asma grave era equivalente em subgrupos com número elevado e reduzido de eosinófilos periféricos ${ }^{32}$.

A possibilidade de utilizar o omalizumab para prevenir a evolução para asma em crianças está a ser avaliada pelo ensaio clínico PARK (Preventing Asthma in High Risk Kids). Neste ensaio, 250 crianças de idade pré-escolar (24-47 meses) com elevado risco de asma (2 a 4 episódios de sibilância no último ano, testes positivos para alergia a aeroalergénios e um familiar em primeiro grau com história de asma ou atopia) receberão omalizumab (ou placebo) durante dois anos e, dois anos após suspensão da terapêutica, será avaliada a prevalência de asma ativa, a gravidade da asma, o número de episódios de sibilância nos doentes e o número de novas sensibilizações alérgicas. Este ensaio de fase 2 tem data estimada de conclusão a 27 de novembro de $2025^{33}$.

\section{TERAPÊUTICA ANTI-IL-5 E ANTI-IL-5RA}

Atualmente, para o tratamento de asma eosinofílica em adultos estão comercializados em Portugal três anticorpos monoclonais dirigidos à IL-5 ou ao seu recetor (IL-5Ra): o mepolizumab, o reslizumab e o benralizumab ${ }^{20}$. Destes, apenas o mepolizumab está aprovado na Europa para idades inferiores a 18 anos.

\section{Mepolizumab}

O mepolizumab é um anticorpo monoclonal humanizado ( $\lg G \mid K)$ dirigido à IL-5 que bloqueia a sua ligação à cadeia alfa do recetor da IL- $5^{34}$. Foi aprovado pela FDA e pela EMA em 2015 para o tratamento de asma eosinofílica grave em adultos e adolescentes com idade igual ou superior a 12 anos $^{35}$. A EMA aprovou em agosto de 2018 a utilização do mepolizumab para crianças a partir dos 6 anos $^{35}$.

Vários ensaios clínicos randomizados de fase III, duplamente cegos, controlados por placebo, multicêntricos, com mepolizumab, incluíram adultos e adolescentes com idades superiores a 12 anos. Contudo, foi incluído um número reduzido de doentes pediátricos (cerca de 30 numa amostra superior a 800 doentes, correspondendo a $2-4 \%$ do total), nenhum dos ensaios se dedicou à avaliação exclusiva desta faixa etária e não foram descritas subanálises desta população ${ }^{34}$, o que limita a extrapolação de dados.

Apesar do número reduzido de adolescentes com idade igual ou superior a 12 anos e da ausência de estudos em crianças com idades entre os 6 e os II anos, a EMA aprovou em agosto de 2018 a utilização do mepolizumab para crianças a partir dos 6 anos $^{35}$. A extensão da indi- 
cação a idades inferiores foi baseada na existência de dados dos ensaios fase 3 em doentes com mais de 12 anos, na semelhança fisiopatológica da asma nos grupos etários 6-II anos e $\geq 12$ anos, no perfil de segurança semelhante nos grupos etários $6-11$ anos e $\geq 12$ anos, na consistência terapêutica e na consistência de mecanismos de ação do mepolizumab nas várias idades ${ }^{35}$.

Entre os estudos realizados incluem-se o DREAM (que incluiu 62I doentes com idades entre os 12 e os 74 anos ${ }^{36}$ ), o MENSA (que incluiu 576 doentes com idades entre os 12 e os 82 anos $^{37}$ ), o SIRIUS (que incluiu 135 doentes com idades entre os 16 e os 74 anos, diagnóstico de asma eosinofílica grave com necessidade de terapêutica diária com glucocorticoide oral, para além de terapêutica com corticoide inalado em altas $\operatorname{doses}^{38}$ ), ○ COSMOS (extensão de 52 semanas dos estudos MENSA e SIRIUS, incluindo 651 doentes, dos quais 414 tinham sido tratados previamente com mepolizumab e $237 \mathrm{com}$ placebo $^{39}$, e o COLUMBA (extensão open-label do DREAM que incluiu 347 doentes que continuaram tratamento durante uma média de 3,5 anos - máximo de $4,5 \operatorname{anos}^{40}$ ). Os detalhes de cada um destes ensaios estão descritos em artigo recente desta Revista ${ }^{20}$.

Em 2019, foi publicado um pequeno ensaio clínico open-label, não controlado, para avaliar a eficácia, segurança e farmacodinâmica do mepolizumab em crianças com asma eosinofílica grave com idades entre os 6 e os II anos $^{41}$. O ensaio incluiu 30 crianças tratadas durante 52 semanas com $40 \mathrm{mg}$ ou $100 \mathrm{mg}$ de mepolizumab, de acordo com o peso inferior ou superior a $40 \mathrm{~kg}$. Quando comparado com o baseline, o tratamento com mepolizumab reduziu o número de eosinófilos circulantes (para cerca de $15,5 \%$ do valor basal entre as 12 e as 52 semanas), reduziu o número de exacerbações $(80 \%$ das crianças tiveram uma redução de pelo menos $50 \%$ ) e melhorou o controlo sintomático (entre outros, uma redução média de $66 \%$ no score ACQ-7 entre as 12 e as 52 semanas). Cerca de $90 \%$ das crianças desenvolveram eventos adversos ( $23 \%$ tiveram eventos adversos graves mas nenhum foi relacionado com o tratamento, de acor- do com os investigadores, tendo resolvido sem a suspensão do mesmo). Não foram detetados anticorpos anti-mepolizumab ${ }^{4}$.

Em Portugal, o mepolizumab está comercializado desde finais de 2016, estando atualmente indicado como tratamento adjuvante da asma eosinofílica refratária grave em adultos, adolescentes e crianças com 6 ou mais anos. A dose recomendada é de $100 \mathrm{mg}$ para adultos e adolescentes com 12 ou mais anos, e $40 \mathrm{mg}$ para crianças entre 6 e II anos, administrados por via subcutânea uma vez a cada 4 semanas. As reações adversas mais frequentemente notificadas durante o tratamento foram cefaleias, reações no local de administração e dorsalgia ${ }^{35}$.

\section{Reslizumab}

O reslizumab é um anticorpo monoclonal humanizado (lgG4K), que, tal como o mepolizumab, é dirigido à IL-5, inibindo a sua atividade ${ }^{20}$. Ao contrário do mepolizumab, que é administrado por via subcutânea, o reslizumab é administrado por via endovenosa e a dose ajustada ao peso ( $3 \mathrm{mg} / \mathrm{kg})$, administrado a cada 4 semanas $^{42}$.

O programa BREATH incluiu quatro ensaios clínicos com reslizumab, randomizados, de fase 3 , duplamente cegos, controlados por placebo, multicêntricos, que incluíram adultos e adolescentes com idades superiores a II anos (embora nenhum dos ensaios tenha incluído apenas doentes de idade pediátrica ou tenham sido descritas subanálises desta população) ${ }^{34}$. Os detalhes e resultados de cada um destes ensaios estão descritos em outras revisões sobre reslizumab ${ }^{43}$ e em artigo recente desta Revista ${ }^{20}$.

No total, os vários ensaios de fase 3 com reslizumab incluíram 39 adolescentes asmáticos ( 12 a 17 anos), que foram aleatorizados para o reslizumab nas doses $0,3 \mathrm{mg} / \mathrm{kg}$ e $3 \mathrm{mg} / \mathrm{kg}$ ou placebo. O perfil de segurança foi semelhante ao placebo mas, em termos de eficácia, o reslizumab não reduziu o número de exacerbações da asma neste grupo etário (razão entre as taxas de exacerbações da asma [reslizumab/placebo] de 2,09$)^{43}$. 
Pedro Botelho Alves, lolanda Alen Coutinho, Ana Luísa Moura, Frederico S. Regateiro

Dado o pequeno tamanho da amostra e os desequilíbrios iniciais resultantes da análise de subgrupos, não se podem tirar conclusões sobre a eficácia do reslizumab na população pediátrica ${ }^{43}$.

O reslizumab foi aprovado pela FDA e pela EMA em 2016 como tratamento de manutenção add-on em adultos com diagnóstico de asma eosinofílica grave ${ }^{34}$. Em Portugal está aprovado desde $2018^{42}$. Até ao momento não houve extensão da indicação do reslizumab a crianças ou adolescentes, e não temos conhecimento de novos ensaios clínicos a decorrer com reslizumab dirigidos à idade pediátrica.

\section{Benralizumab}

O benralizumab é um anticorpo monoclonal humanizado $(\mathrm{lgG} \mid \mathrm{K})$ que se liga à subunidade alfa do recetor da IL-5 (IL-5Ra, CDI25) ${ }^{20}$, resultando na apoptose das células que expressam IL-5Ra, sobretudo eosinófilos e basófilos, através da citotoxicidade mediada por células dependentes de anticorpo (ADCC antibody-dependent cell-mediated cytotoxicity) $)^{34}$.

Dois ensaios clínicos de fase 3 realizados com o benralizumab incluíram adultos e adolescentes com idades superiores a 12 anos - os estudos SIROCCO e CALIMA ${ }^{34,44}$.

Nestes estudos foram incluídos 108 adolescentes asmáticos (sob terapêutica com altas doses de corticoide inalado associado a broncodilatador de longa ação e com duas ou mais exacerbações no ano anterior), com idades entre os 12 e os 17 anos, dos quais 46 receberam placebo, 40 receberam benralizumab a cada 4 semanas nas primeiras 3 doses, e depois a cada 8 semanas, e 22 receberam benralizumab a cada 4 semanas $^{45}$. A taxa de exacerbação da asma em adolescentes que receberam tratamento foi de 0,70 ( $n=40$, IC 95\%: 0,42; I, I8) comparado com 0,4I para o placebo ( $n=46, I C$ 95\%: 0,23; $0,73)^{42}$. Dado o pequeno tamanho da amostra e os desequilíbrios iniciais das amostras não é possível tirar conclusões sobre a eficácia do benralizumab na asma na população pediátrica ${ }^{45}$. As extensões BORA/MELTEMI, atualmente em desenvolvimento, poderão trazer mais informações sobre a eficácia e a segurança do benralizumab em adolescentes ${ }^{46}$.

O benralizumab foi aprovado pela FDA em 2017 como tratamento de manutenção add-on em doentes com diagnóstico de asma eosinofílica grave para idades iguais ou superiores a 12 anos e pela EMA em 2018 em adultos ${ }^{45}$. Em Portugal teve aprovação de entrada no mercado em 2019. A dose recomendada de benralizumab é $30 \mathrm{mg}$ administrados por injeção subcutânea a cada 4 semanas para as primeiras 3 doses, e depois a cada 8 semanas. As reações adversas mais frequentemente notificadas durante o tratamento foram cefaleia e faringite ${ }^{45}$.

\section{TERAPÊUTICA ANTI-IL-4/IL-I3}

\section{Dupilumab}

A IL-4 e IL-I3 estão envolvidas não só na fisiopatologia da asma, como também na fisiopatologia de outras patologias atópicas, como a dermatite atópica e a rinossinusite crónica.

O dupilumab é um anticorpo monoclonal humano do tipo $\operatorname{lgG} 4$ que se liga à subunidade $\alpha$ do recetor da interleucina-4 (IL-4Ra), bloqueando a sinalização das interleucinas IL-4 e IL-I3 e inibindo respostas Th $2^{47}$.

O dupilumab foi aprovado para o tratamento da dermatite atópica moderada a grave, em adultos no ano de $2017^{48}$ e em adolescentes em 2019. Em 2018 foi aprovado pela FDA e em 2019 pela EMA como tratamento adjuvante de manutenção em adultos e adolescentes acima dos 12 anos com asma grave e endótipo Th2 caraterizado por aumento dos eosinófilos no sangue e/ou aumento do $\mathrm{FeNO}^{49}$. Em setembro de 2019 foi também aprovado para o tratamento da rinossinusite crónica grave com polipose nasal ${ }^{49}$. $O$ dupilumab é administrado por via subcutânea numa dose inicial de $400 \mathrm{mg}$, seguida de $200 \mathrm{mg}$ administradas em semanas alternadas (em doentes com asma grave sob corticoterapia oral, a dose inicial é de $600 \mathrm{mg}$ e as subsequentes de $300 \mathrm{mg}$ ).

Vários estudos avaliaram a eficácia e a segurança do dupilumab no tratamento da asma grave em adultos e 
adolescentes $^{48}$. O estudo QUEST, publicado em 2018, incluiu 1902 doentes com asma grave e idade igual ou superior a 12 anos (média de idades de 47,9 anos), incluindo um total de 107 adolescentes. Este estudo avaliou o efeito do tratamento com dupilumab administrado de 2 em 2 semanas na dose de $200 \mathrm{mg}$ ou $300 \mathrm{mg}$, durante um período de 56 semanas. $O$ dupilumab reduziu significativamente a frequência de exacerbações de asma (redução da taxa anualizada de exacerbações de $65,8 \%$ vs. placebo), tendo havido também uma melhoria da função pulmonar (às 12 semanas de tratamento verificou-se um aumento

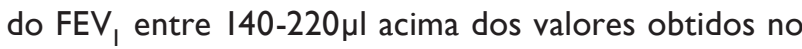
grupo placebo) ${ }^{47}$. O estudo VENTURE incluiu 210 doentes com idade igual ou superior a 12 anos (média de idades de 5I,3 anos) com asma grave sob corticoterapia sistémica. $O$ estudo avaliou a eficácia do tratamento com dupilumab durante um período de 24 semanas, tendo como outcome primário a variação da dose diária de corticosteroide sistémico necessária para controlo da doença. Os doentes que receberam dupilumab apresentaram uma redução de $70,1 \%$ da dose de corticoide sistémico versus redução de $41,9 \%$ no grupo placebo ${ }^{50,51}$.

Nos doentes em tratamento com dupilumab para a dermatite atópica foi observado um aumento da frequência de conjuntivite de várias etiologias, e outros distúrbios oculares, como queratite, para além de reações no local da injeção ${ }^{21,49}$. Nos ensaios clínicos para asma, a reação adversa mais frequente foi eritema no local de injeção. Foram notificados casos muito raros de reação anafilática no programa de desenvolvimento da asma ${ }^{49}$.

Relativamente à faixa etária das crianças com idades compreendidas entre os 6 e os 12 anos está a decorrer um ensaio clínico de fase 3 , randomizado e controlado com placebo, que inclui 402 crianças - o estudo VOYAGE -, cujo objetivo primário é avaliar a eficácia do dupilumab em doentes com asma persistente não controlada, através da comparação da taxa anual de exacerbação durante o período de tratamento com dupilumab versus placebo. Este ensaio clínico tem como data prevista de conclusão o mês de novembro de $2020^{52}$.

\section{TERAPÊUTICA ANTI-TSLP}

A linfopoetina do estroma tímico (TSLP), uma alarmina produzida pelas células epiteliais em resposta a estímulos pró-inflamatórios, induz a produção de citocinas Th2 em células dendríticas e ILC2. Os níveis de TSLP encontram-se aumentados nas vias aéreas dos doentes com asma ${ }^{21}$. Atualmente encontram-se em desenvolvimento vários anticorpos monoclonais anti-TSLP, estando em fases mais avançadas o tezepelumab (fase 3$)^{53}$ e o CSJII7 (fase 2, ainda sem ensaios na idade pediátrica, mas que tem a particularidade interessante de poder ser administrado por via inalatória) ${ }^{54}$.

\section{Tezepelumab}

O tezepelumab é um anticorpo monoclonal humano do tipo lgG2 de administração subcutânea. Vários ensaios clínicos têm avaliado a eficácia do tezepelumab em adultos, não existindo ainda dados sobre a sua segurança e eficácia em crianças e adolescentes ${ }^{55}$.

O ensaio NAVIGATOR ${ }^{53}$, um ensaio clínico randomizado de fase 3, controlado com grupo placebo, teve como objetivo principal avaliar a eficácia e a segurança do tezepelumab em adultos e adolescentes com 12 ou mais anos e com asma grave. A duração do ensaio foi de 52 semanas e atingiu o outcome primário com uma redução estatisticamente significativa e clinicamente significativa da taxa anual de exacerbações.

\section{CONCLUSÃO}

Os ensaios clínicos e estudos de vida real são promissores relativamente à eficácia dos anticorpos monoclonais na asma grave associados a um bom perfil de segurança em idades pediátricas. Nas crianças e adolescentes, as recentes aprovações de biológicos que atuam nas vias da IL-5 (mepolizumab) e da IL-4/IL-I3 (dupilumab) vieram alargar o espectro de alvos terapêuticos para além da lgE (omalizumab, que já se encontra comercializado para idades pediátricas há cerca de 10 anos). 
De uma forma geral podemos dizer que os ensaios clínicos publicados em asma grave pediátrica têm uma duração curta ( $\mathrm{l}$ a 2 anos, alguns deles com extensões de mais 2-3 anos), avaliando apenas outcomes incluídos no período do estudo e, excetuando o omalizumab, foram conduzidos num reduzido número de doentes, com muitos dos resultados a serem extrapolados a partir de grupos maioritariamente constituídos por adultos. Seria desejável que, sobretudo em crianças e adolescentes, os resultados fossem avaliados em períodos mais longos, inclusive até à idade adulta, e que incluíssem avaliações do desenvolvimento pulmonar durante o crescimento, para além dos critérios habituais (exacerbações, exacerbações graves, scores de qualidade de vida, etc.). É previsível que o melhor controlo da asma durante a infância e adolescência permita também melhorias colaterais muito importantes nestas faixas etárias, por exemplo no crescimento estatoponderal, na performance escolar, na capacidade de fazer exercício físico e desporto, na integração social e em outros parâmetros da qualidade de vida e crescimento integral.

\section{Conflito de interesses}

Este trabalho não recebeu quaisquer apoios financeiros para a sua realização. Frederico $S$. Regateiro declara ter recebido apoio financeiro como palestrante ou consultor científico de AstraZeneca, Novartis, GlaxoSmithKline, TEVA, Sanofi e Lusomedicamenta.

\section{Contacto:}

Frederico S. Regateiro

Serviço de Imunoalergologia

Centro Hospitalar Universitário de Coimbra

Praceta Prof. Mota Pinto

3000-075 Coimbra, Portugal

Email: regateiro@gmail.com

\section{REFERÊNCIAS}

I. Maglione M, Poeta M, Santamaria F. New drugs for pediatric asthma. Front Pediatr 2018; 6: 432.

2. Papadopoulos NG, Arakawa H, Carlsen K.-H, Custovic A, Gern J, Lemanske R, Le Souef P, et al. International consensus on (ICON) pediatric asthma. Allergy 2012; 67: 976-97.

3. Couto M, Santos $A$. Consenso internacional em asma pediátrica (ICON). Rev Port Imunoalergologia 2014; 22: 231 -9.

4. Pité H, Pereira AM, Gaspar A, Morais-Almeida M. Fenótipos de sibilância em idade pré-escolar e relação com persistência de asma até à adolescência. Rev Port Imunoalergologia 2015; 23: 159-74.

5. Phelan PD, Robertson CF, Olinsky A. The Melbourne asthma study: 1964-1999. J Allergy Clin Immunol 2002; 109:189-94.

6. Turner SW, Palmer LJ, Rye PJ, Gibson NA, Judge PK, Cox M, Young $\mathrm{S}$, et al. The relationship between infant airway function, childhood airway responsiveness, and asthma. Am J Respir Crit Care Med 2004;169:921-7.

7. Palmer LJ, Rye PJ, Gibson NA, Burton PR, Landau LI, Lesouëf PN. Airway responsiveness in early infancy predicts asthma, lung function, and respiratory symptoms by school age. Am J Respir Crit Care Med 200I; 163: 37-42.

8. Martinez FD, Wright AL, Taussig LM, Holberg CJ, Halonen M, Morgan WJ. Asthma and wheezing in the first six years of life. The Group Health Medical Associates. N Engl J Med 1995; 332:133-8.

9. Wenzel SE. Asthma phenotypes: the evolution from clinical to molecular approaches. Nat Med 2012;18:716-25.

10. Svenningsen S, Nair P. Asthma endotypes and an overview of targeted therapy for asthma. Front Med 2017;4:158.

II. Just J, Deschildre A, Lejeune S, Amat F. New perspectives of childhood asthma treatment with biologics. Pediatr Allergy Immunol 2019; 30:159-71.

12. Fitzpatrick AM, Baena-Cagnani CE, Bacharier LB. Severe asthma in childhood: recent advances in phenotyping and pathogenesis. Curr Opin Allergy Clin Immunol 2012; 12: 193-20I.

13. Fitzpatrick AM, Moore WC. Severe Asthma Phenotypes - How should they guide evaluation and treatment? J Allergy Clin Immunol: In Practice 2017;5:90I-8

14. Global Initiative for Asthma. Global Strategy for Asthma Management and Prevention, 2020. Available from: https://ginasthma.

\section{Acrónimos e abreviaturas}

FeNO, fração exalada de óxido nítrico; FEVI, forced expiratory volume in one second; ICS, corticosteroide inalado; IL, interleucina; ILC-2, células linfoides inatas tipo 2; INFARMED, Infarmed - Autoridade Nacional do Medicamento e Produtos de Saúde, IP; IV, endovenosa; LABA, agonista $\beta$-adrenérgico de longa ação; SC, subcutânea; Th, T-helper; TNF, tumour necrosis factor; TSLP, thymic stromal lymphopoietin 
org/wp -content/uploads/2020/04/GINA -2020 -full -report_final_wms.pdf (accessed April 15 2020).

15. Lang A, Carlsen KH, Haaland G, Devulapalli CS, Munthe-Kaas M, Mowinckel P, et al. Severe asthma in childhood: assessed in 10 year olds in a birth cohort study. Allergy 2008; 63:1054-60.

16. Nordlund B, Melén E, Schultz ES, Grönlund H, Hedlin G, Kull I. Prevalence of severe childhood asthma according to the WHO. Respir Med 2014;108:1234-7.

17. Allen DB. Safety of inhaled corticosteroids in children. Pediatr Pulmonol 2002; 33: 208-20.

18. Simons FE. Benefits and risks of inhaled glucocorticoids in children with persistent asthma. J Allergy Clin Immunol 1998; 102:S77-84.

19. Lanz MJ, Gilbert I, Szefler SJ, Murphy KR. Can early intervention in pediatric asthma improve long-term outcomes? A question that needs an answer. Pediatr Pulmonol 2019;54:348-57.

20. Regateiro F, Moura AL, Faria E. Novos biológicos para o tratamento da asma. Rev Port Imunoalergologia 2017;25:99-1/3.

21. Abrams EM, Becker AB, Szefler SJ. Current state and future of biologic therapies in the treatment of asthma in children. Pediatr Allergy Immunol Pulmonol 2018;31:119-31.

22. Schulman ES. Development of a monoclonal anti-immunoglobulin E antibody (omalizumab) for the treatment of allergic respiratory disorders. Am J Respir Crit Care Med 200I;164:S6-II.

23. U.S. Food and Drug Administration - Xolair. Available at: https:// www.fda.gov/ForConsumers/ConsumerUpdates/ucm I03291.html (accessed February 13 2020)

24. European Medicines Agency - Xolair. Available at: https://www. ema.europa.eu/en/medicines/human/paediatric-investigation-plans/emea-000735-pip0I-09 (accessed February 15 2020)

25. Limb SL, Starke PR, Lee CE, Chowdhury BA. Delayed onset and protracted progression of anaphylaxis after omalizumab administration in patients with asthma. J Allergy Clin Immunol 2007;120:I378-8I.

26. Busse WW, Morgan WJ, Gergen PJ, Mitchell HE, Gern JE, Liu $\mathrm{AH}$, et al. Randomized trial of omalizumab (anti-lgE) for asthma in inner-city children. N Engl J Med 20II;364: 1005-15.

27. Lanier B, Bridges T, Kulus M, Taylor AF, Berhane I, Vidaurre CF. Omalizumab for the treatment of exacerbations in children with inadequately controlled allergic (lgE-mediated) asthma. J Allergy Clin Immunol 2009;124:1210-6.

28. Milgrom H, Berger W, Nayak A, Gupta N, Pollard S, McAlary M, et al. Treatment of childhood asthma with anti-immunoglobulin E antibody (omalizumab). Pediatrics 200I; 108:E36.

29. Teach SJ, Gill MA, Togias A, Sorkness CA, Arbes SJ, Calatroni A, et al. Preseasonal treatment with either omalizumab or an inhaled corticosteroid boost to prevent fall asthma exacerbations. J Allergy Clin Immunol 20I5; 136:1476-85.

30. Brodlie M, McKean MC, Moss S, Spencer DA. The oral corticosteroid-sparing effect of omalizumab in children with severe asthma. Arch Dis Child 2012;97:604-9.
3I. Deschildre A, Marguet C, Salleron J, Pin I, Rittié JL, Derelle J, et al. Add-on omalizumab in children with severe allergic asthma: a I-year real life survey. Eur Respir J 2013;42: 1224-33.

32. Humbert M, Taillé C, Mala L, Le Gros V, Just J, Molimard M. Omalizumab effectiveness in patients with severe allergic asthma according to blood eosinophil count: the STELLAIR study. Eur Respir J 2018;51:1702523.

33. ClinicalTrials - Preventing asthma in high risk kids (PARK). Available at: https://clinicaltrials.gov/ct2/show/NCT02570984 (accessed February II 2020).

34. Drummond M, Cordeiro CR, Neuparth N. Severe asthma. Ist ed. Barcelona; Permanyer Portugal: 2018.

35. European Medicines Agency - Nucala. Available at: https://www. ema.europa.eu/en/medicines/human/EPAR/nucala (accessed February 13 2020)

36. Pavord ID, Korn S, Howarth P, Bleecker ER, Buhl R, Keene ON, et al. Mepolizumab for severe eosinophilic asthma (DREAM): a multicentre, double-blind, placebo-controlled trial. Lancet 20I2;380: 65I-9.

37. Ortega HG, Yancey SW, Mayer B, Gunsoy NB, Keene ON, Bleecker ER, et al. Mepolizumab treatment in patients with severe eosinophilic asthma. N Engl J Med 2014;37I:II98-207.

38. Bel EH, Wenzel SE, Thompson PJ, Prazma CM, Keene ON, Yancey SW, et al. Oral glucocorticoid-sparing effect of mepolizumab in eosinophilic asthma. N Engl J Med 2014;37I:II89-97.

39. Lugogo N, Domingo C, Chanez P, Leigh R, Gilson MJ, Price RG, et al. Long-term efficacy and safety of mepolizumab in patients with severe eosinophilic asthma: A multi-center, open-label, Phase Illb study. Clin Ther 2016;38:2058-70.el.

40. Khatri S, Moore W, Gibson PG, Leigh R, Bourdin A, Maspero J, et al. Assessment of the long-term safety of mepolizumab and durability of clinical response in patients with severe eosinophilic asthma. J Allergy Clin Immunol 2019;143:1742-5I.

4I. Gupta A, Ikeda M, Geng B, Azmi J, Price RG, Bradford ES, et al. Long-term safety and pharmacodynamics of mepolizumab in children with severe asthma with an eosinophilic phenotype. J Allergy Clin Immunol 2019;144:1336-42.

42. European Medicines Agency - Cinqaero. Available at: https:// www.ema.europa.eu/en/medicines/human/EPAR/cinqaero (accessed February 14 2020)

43. MásperoJ. Reslizumab in the treatment of inadequately controlled asthma in adults and adolescents with elevated blood eosinophils: clinical trial evidence and future prospects. Ther Adv Respir Dis 2017;II:3II-25.

44. Bleecker ER, Fitzgerald JM, Chanez P, Papi A, Weinstein SF, Barker $P$, et al. Efficacy and safety of benralizumab for patients with severe asthma uncontrolled with high-dosage inhaled corticosteroids and long-acting $\beta 2$-agonists (SIROCCO): a randomised, multicentre, placebo-controlled phase 3 trial. Lancet 2016;388:2II5-27. 
Pedro Botelho Alves, Iolanda Alen Coutinho, Ana Luísa Moura, Frederico S. Regateiro

45. European Medicines Agency - Fasenra. Available at: https://www. ema.europa.eu/en/medicines/human/EPAR/fasenra (accessed February 15 2020)

46. Busse WW, Bleecker ER, Fitzgerald JM, Ferguson GT, Barker P, Sproule S, et al. Long-term safety and efficacy of benralizumab in patients with severe, uncontrolled asthma: I-year results from the BORA phase 3 extension trial. Lancet Respir Med 2019; 7:46-59

47. Castro M, Corren J, Pavord ID, Maspero J, Wenzel S, Rabe KF, et al. Dupilumab efficacy and safety in moderate-to-severe uncontrolled asthma. N Engl J Med 2018;378:2486-96.

48. Renert-Yuval Y, Guttman-Yassky E. What's new in atopic dermatitis. Dermatol Clin 2019;37:205-13.

49. European Medicines Agency - Dupixent. Available at: https:// www.ema.europa.eu/en/medicines/human/EPAR/dupixent (accessed February 16 2020).

50. Rabe KF, Nair P, Brusselle G, Maspero JF, Castro M, Sher L, et al. Efficacy and safety of dupilumab in glucocorticoid-dependent severe asthma. N Engl J Med 2018;378:2475-85.
5I. European Medicines Agency - New add-on treatment for patients with severe asthma. Available at: https://www.ema.europa.eu/en/ documents/press-release/press-release-new-add-treatment-patients-severe-asthma_en.pdf (accessed March I 2020)

52. ClinicalTrials - Evaluation of dupilumab in children with uncontrolled asthma (VOYAGE). Available at: https://clinicaltrials.gov/ ct2/show/NCT02948959 (accessed February 2l 2020).

53. ClinicalTrials - Study to evaluate tezepelumab in adults \& adolescents with severe uncontrolled asthma (NAVIGATOR). Available at: https://clinicaltrials.gov/ct2/show/NCT03347279 (accessed March I 2020)

54. ClinicalTrials - A bronchoprovocation study to assess the safety, tolerability, pharmacokinetics and pharmacodynamics of CSJII7 in adult subjects with mild atopic asthma. Available at: https://clinicaltrials.gov/ct2/show/NCT031388II (accessed March I 2020).

55. Corren J, Parnes JR, Wang L, Mo M, Roseti SL, Griffiths JM, et al. Tezepelumab in adults with uncontrolled asthma. N Engl J Med 2017; 377:936-46. 